\title{
Direct Imaging Mass Spectrometry of Plant Leaves Using Surface-assisted Laser Desorption/Ionization with Sputter- deposited Platinum Film
}

\author{
Tomoyuki Ozawa, ${ }^{*}$ Issey Osaka, ${ }^{* * \dagger}$ Satoshi Hamada, $* * *$ Tatsuya Murakami, ${ }^{* *}$ Akio Miyazato, ${ }^{* *}$ \\ Hideya KAWASAKI,* and Ryuichi ARAKAWA *† \\ *Department of Chemistry and Materials Engineering, Faculty of Chemistry, Materials, and Bioengineering, \\ Kansai University, 3-3-35 Yamate-cho, Suita, Osaka 564-8680, Japan \\ **Center for Nano Materials and Technology, Japan Advanced Institute of Science and Technology, \\ 1-1 Asahidai, Nomi, Ishikawa 923-1292, Japan \\ ***Analysis Research Department, Chemical Research Laboratories, Nissan Chemical Industries, Ltd., \\ 10-1 Tsuboi-Nishi 2-chome, Funabashi, Chiba 274-8507, Japan
}

\begin{abstract}
Plant leaves administered with systemic insecticides as agricultural chemicals were analyzed using imaging mass spectrometry (IMS). Matrix-assisted laser desorption/ionization (MALDI) is inadequate for the detection of insecticides on leaves because of the charge-up effect that occurs on the non-conductive surface of the leaves. In this study, surfaceassisted laser desorption/ionization with a sputter-deposited platinum film (Pt-SALDI) was used for direct analysis of chemicals in plant leaves. Sputter-deposited platinum (Pt) films were prepared on leaves administered with the insecticides. A sputter-deposited Pt film with porous structure was used as the matrix for Pt-SALDI. Acephate and acetamiprid contained in the insecticides on the leaves could be detected using Pt-SALDI-MS, but these chemical components could not be adequately detected using MALDI-MS because of the charge-up effect. Enhancement of ion yields for the insecticides was achieved using Pt-SALDI, accompanied by prevention of the charge-up effect by the conductive Pt film. The movement of systemic insecticides in plants could be observed clearly using Pt-SALDI-IMS. The distribution and movement of components of systemic insecticides on leaves could be analyzed directly using Pt-SALDI-IMS. Additionally, changes in the properties of the chemicals with time, as an indicator of the permeability of the insecticides, could be evaluated.
\end{abstract}

Keywords Pt film, imaging mass spectrometry (IMS), surface-assisted laser desorption ionization (SALDI), leaf, agricultural chemicals

(Received November 14, 2015; Accepted January 29, 2016; Published May 10, 2016)

\section{Introduction}

Development of techniques for imaging agricultural chemicals in plants are important for observing the movement of systemic insecticides and their metabolites. Most qualitative and quantitative analyses of drugs and agricultural chemicals have utilized liquid chromatography mass spectrometry (LC-MS). ${ }^{1-3}$ However, LC-MS does not facilitate imaging analysis. Generally, the movement of agricultural chemicals in plants can be observed by high resolution radioisotope imaging, ${ }^{4}$ although it is difficult to handle expensive and dangerous radioisotopes. Radioisotope experiments require expensive and exclusive facilities for prevention of exposure to radiation. Therefore, simple and inexpensive new methods for imaging plants are required in the pharmaceutical and agricultural fields to examine the movement of systemic insecticides and their metabolites in plants. Müller et al. have reported the imaging of plant by

† To whom correspondence should be addressed.

E-mail: o-issey@jaist.ac.jp; arak@kansai-u.ac.jp desorption electrospray ionization mass spectrometry with imprint. $^{5}$ However, it was difficult to achieve homogeneous transfers of various compounds in the imprinting process. Therefore, a new direct and simple imaging method is required. Recently, matrix-assisted laser desorption/ionization mass spectrometry (MALDI) imaging mass spectrometry (IMS) was developed as a technique for detection of analytes and for visualizing the distribution of molecules in a dissected biological tissue on a target plate. ${ }^{6,7}$ MALDI with UV-absorbing organic matrices has been widely used for the analysis of biomolecules. ${ }^{8-10} \quad \alpha$-Cyano-4-hydroxycinnamic acid (CHCA) and 2,5-dihydroxybenzoic acid (DHB) are the most common MALDI matrices used for the ionization of various compounds such as peptides and lipids. ${ }^{11-13}$ However, characterization of small molecules via MALDI-MS is difficult because of interference and detector saturation by excess matrix ions, which are inevitable in the MALDI analysis of small molecules such as environmental and agricultural chemicals. ${ }^{14,15}$ In MALDI-IMS, an ITO film is deposited on the surface of the target plate for charge separation of ions produced by MALDI. The tissue section must be prepared at a thickness of $10-50 \mu \mathrm{m}$ 
to maintain the conductivity of the ITO surface. MALDI-IMS of thick tissue such as plant leaves could not be achieved due to charge-up in the MALDI ion source. ${ }^{16}$ The charge-up effect of the ion source causes low resolution of the mass spectra. Therefore, a conductive surface is necessary to obtain high quality mass spectra in MALDI.

As organic-matrix-free approaches to MALDI, surfaceassisted laser desorption/ionization (SALDI) has been investigated for the detection of small molecules. ${ }^{17-23}$ In the SALDI with metal nanoparticles and nanostructured substrates such as Au, Ag and porous silicon have been utilized. The potential ability of the nanoparticles for SALDI matrices has been investigated to achieve high ion yields of pigments and saccharides. ${ }^{19-23}$ Although the Au nanoparticle is widely used as SALDI matrix, strong intensities of Au clusters appeared as interference peaks in the mass spectrum of the SALDI with larger sized $\mathrm{Au}$ nanoparticles. Therefore, smaller sized $\mathrm{Au}$ nanoparticles were suitable as Au-SALDI matrix. ${ }^{24}$ In the case of Ag-SALDI, the aromatic compounds can be selectively detected as $\mathrm{Ag}$ adduct form. ${ }^{25}$ In the case of Pt-SALDI, phospholipids and peptides could be detected using $\mathrm{Pt}$ nanoflower matrix. ${ }^{26}$ However, various techniques were required for synthesis of these nanomaterials. On the other hand, metal film as SALDI matrix could be prepared rapidly and simply. The present group and Dufresne et al. respectively reported new SALDI methods using Pt and Ag films prepared on the surface of the sample..$^{27-30}$ It was found that the Pt film gave higher ion yields of polymer and diacylglycerol without modification and alloying. Sputter-deposited Pt films with pores were formed on the dried sample prior to sputtering. ${ }^{27,28}$ SALDI with sputterdeposited $\mathrm{Pt}$ film allows IMS analysis of non-conductive materials without the need for extraction. ${ }^{27}$ In this study, the distribution of agricultural chemicals in thick and non-conductive leaves is investigated. Cross section of soybean cotyledon was analyzed by MALDI-IMS ${ }^{31}$ and oligosaccharide in garlic was analyzed by SALDI-IMS. ${ }^{32}$ However, the direct imaging analysis of leaves could not be achieved. In this study, non-conductive leaves picked from a plant grown in soil mixed with the same solution were prepared and analyzed directly using Pt-SALDI-IMS. Analytical technique for the direct analysis of distribution and movement of the chemicals in plant leaves using Pt-SALDI-IMS without using radioisotope are described here. It is conceivable that Pt-SALDI allows for the imaging mass analysis of various samples such as plant tissue, biological tissue, paper, and film because of the conductivity.

\section{Experimental}

\section{Reagents and chemicals}

Methanol and acetonitrile (AcCN) were purchased from Wako Pure Chemicals (Osaka, Japan). The matrix substance for MALDI-MS (2,5-dihydroxybenzoic acid) was purchased from Sigma-Aldrich (MO, USA). All chemicals were of analytical grade and were used without further purification. Agrochemicals (pesticides and fungicides) were purchased from Sumitomo Chemical Garden Products Inc., Tokyo, Japan.

\section{Sample preparation}

Acephate and acetamiprid, which are components of agricultural chemicals and were used as standard samples, were prepared at concentrations of $3.1,12.5$, and $50 \mathrm{ng} / \mathrm{mL}$ by dilution with water. A synthetic polymer, polyethylene glycol (PEG), was dissolved in methanol and prepared at a concentration of $50 \mathrm{ng} / \mathrm{mL}$. A $1.0-\mu \mathrm{L}$ aliquot of each solution was applied to
Pansy leaf samples (Viola $x$ wittrockiana) and dried.

A horticultural chemical agent, GF Orutoran $(1 \mathrm{~g})$, containing acephate as a vermicide at a concentration of $5 \%$ in minerals was mixed into the soil in which an Ivy (Hedera) was planted. The leaves growing at a distance of $30 \mathrm{~cm}$ from the soil were picked off from the plant after $4,8,11$, and 14 days, respectively. The all prepared leaves were stuck with doubles-sided tape on slide glass for MALDI-IMS. All prepared leaves were analyzed using MALDI- and Pt-SALDI-IMS.

\section{Imaging mass spectrometry}

For the Pt-SALDI experiment, Pt was deposited on the leaves with standard samples or agricultural chemicals by using a commercially available magnetron sputtering device (E-1030, HITACHI, Tokyo, Japan) at a discharge current of $20 \mathrm{~mA}$ under reduced pressure $(5 \mathrm{~Pa})$ at $4^{\circ} \mathrm{C}$. Pt films with thicknesses of $10 \mathrm{~nm}$ (established from the film growth rate) were formed to deposit on the leaves with the compounds. For the MALDIIMS experiment, matrix solutions were applied to the leaves with the compounds using an automatic matrix sprayer (TMsprayer; HTX Technologies, NC, USA) under the following conditions: lock-heated temperature: $75^{\circ} \mathrm{C}$, nozzle velocity: $1000 \mathrm{~mm} / \mathrm{min}$, number of passes: 16 , flow rate: $120 \mu \mathrm{l} / \mathrm{min}$. 2,5-Dihydroxybenzoic acid (DHB) solution prepared using $\mathrm{H}_{2} \mathrm{O} / \mathrm{AcCN}$ (1/1) with $0.1 \%$ TFA at a concentration of $5 \mathrm{mg} / \mathrm{mL}$ was used as a matrix solution. The ion yields of the samples obtained with Pt-SALDI were compared with that obtained using MALDI and were further evaluated.

Imaging mass spectrometry (IMS) experiments were performed via matrix-assisted laser desorption/ionization timeof-flight mass spectrometry (MALDI-TOFMS) using an AutoFlex III mass spectrometer (Bruker Daltonics Inc., Bremen, Germany) equipped with a Nd:YAG laser $(355 \mathrm{~nm})$. All spectra were recorded in the positive ion reflectron mode. The spot diameter of the laser was $100 \mu \mathrm{m}$. The step sizes for the raster pattern between adjacent laser firing points in the imaging experiments were set to $150 \mu \mathrm{m}$ for analysis of the leaves with GF Orutoran, and those to which the agricultural chemical standards were applied. The acceleration voltage and reflectron voltage 1 were both $21 \mathrm{kV}$. Reflectron voltage 2 was set to $9.7 \mathrm{kV}$.

\section{Results and Discussion}

Analysis of standard samples on leaves

Recently, the distribution of metabolites in plants was investigated using MALDI-IMS; however, difficult sample preparation by slicing the plant tissue thinly and spraying matrix solution homogeneously on the tissue sections was required. ${ }^{16}$ For MALDI-IMS, the samples must be thin sections of less than $30 \mu \mathrm{m}$ or have conductive surfaces. Therefore, direct imaging analysis of plant leaves is difficult because of the non-conductive surface of the leaf, which could not be sliced horizontally. To directly evaluate the permeability of agricultural chemicals into plants with time without using a radioisotope, the distribution of chemicals on the leaves was analyzed using Pt-SALDI-IMS.

In this experiment, leaves of Pansy spotted with standard solutions of agricultural chemicals and a synthetic polymer such as acephate, acetamiprid, and PEG were analyzed. The ion yields of the standard samples obtained with Pt-SALDI were compared with those obtained with MALDI to evaluate the influence of the charge-up effect on the non-conductive surface of the leaves. MALDI and Pt-SALDI spectra of the sample distribution on the leaves are shown in Fig. 1. Sodium and 

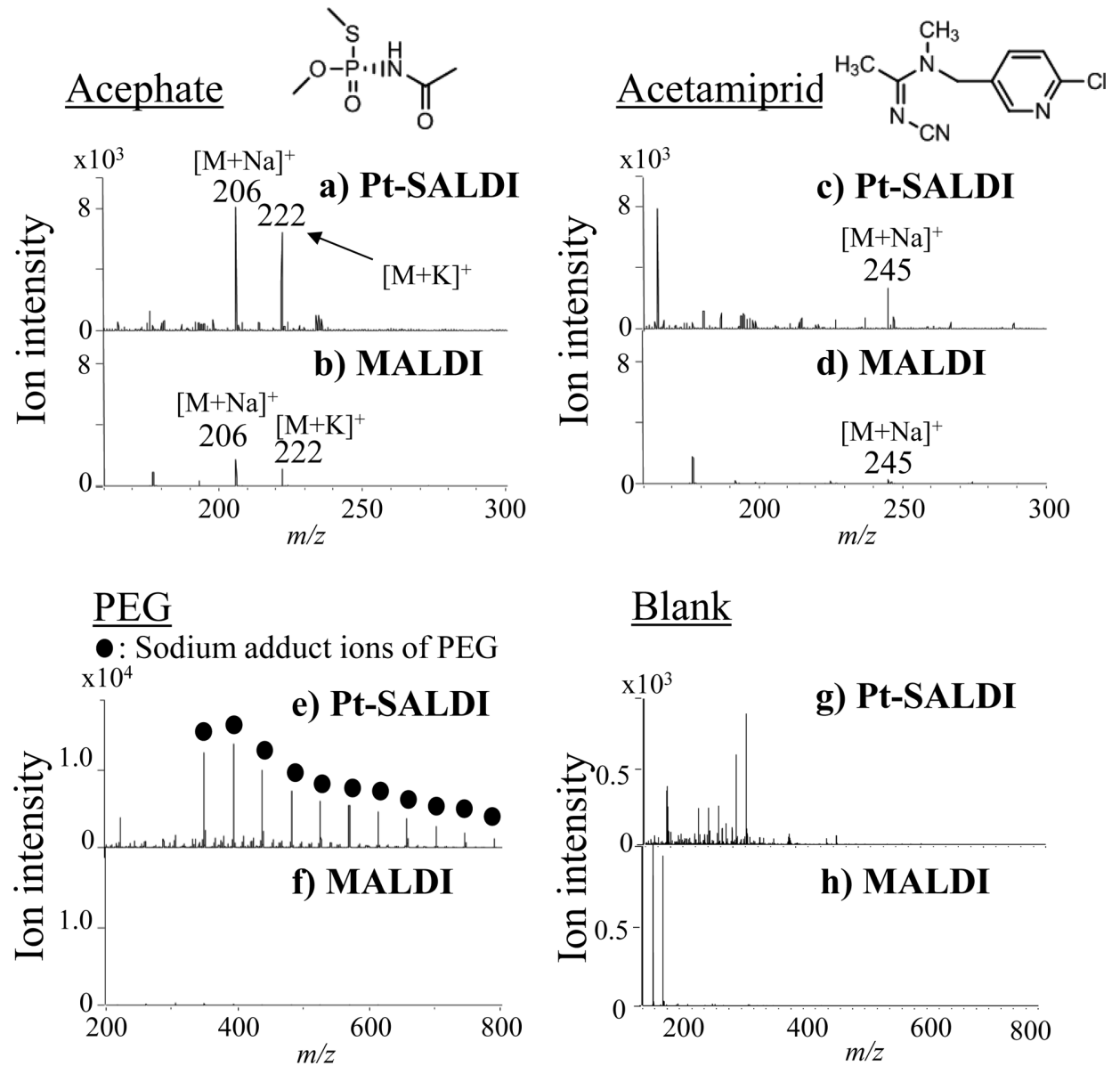

Fig. 1 Mass spectra of $(a-b)$ acephate, $(c-d)$ acetamiprid, $(e-f)$ PEG, $(g-h)$ blank on leaves of Pansy using (a, c, e, g) Pt-SALDI and (b, d, f, h) MALDI.

potassium adduct ions of acephate at $\mathrm{m} / \mathrm{z}, 206$ and $\mathrm{m} / \mathrm{z}, 222$ in Figs. $1 \mathrm{a}$ and $1 \mathrm{~b}$ and the sodium adduct ion of acetamiprid at $\mathrm{m} / \mathrm{z}$ 245 in Figs. 1c and 1d were detected with both ionization methods. Sodium adduct ions of PEG were observed with Pt-SALDI only as shown in Fig. 1e. Pt-SALDI produced high ion yields of acephate and acetamiprid ions, in contrast to the low ion yields of these ions obtained with MALDI. In the MALDI process, these sample ions were generated from the surface of the leaves by using a UV pulse laser. A plume of the MALDI matrix, generated by the laser irradiation, could cause slight desorption of the sample and MALDI matrix ions on the non-conductive surface of the leaves. However, most of these ions were not introduced into the mass spectrometer because of the non-conductivity. During Pt-SALDI, these sample ions could be effectively desorbed and detected with intense signals because of the conductivity of the Pt-film surface. It is conceivable that the charge-up effect resulted in the low ion yields in MALDI. The results indicated that Pt-SALDI with no charge-up effect has potential for the direct analysis of leaves. In Figs. 1g - 1h, the $m / z, 206$ and 222, 245 ions and PEG ions were not observed on the surface of the leaf. The obtained ion yields of the standard samples on the Pansy are summarized in Fig. 2. The signal-to-noise ratios $(S / N)$ of the ion peaks for acephate and acetamiprid obtained by Pt-SALDI were 2.5, 44 times higher than obtained with MALDI. The enrichment of the ion yields for the standard samples and prevention of the chargeup effect by using the conductive Pt film was achieved with the Pt-SALDI technique. It should be noted that the optimized

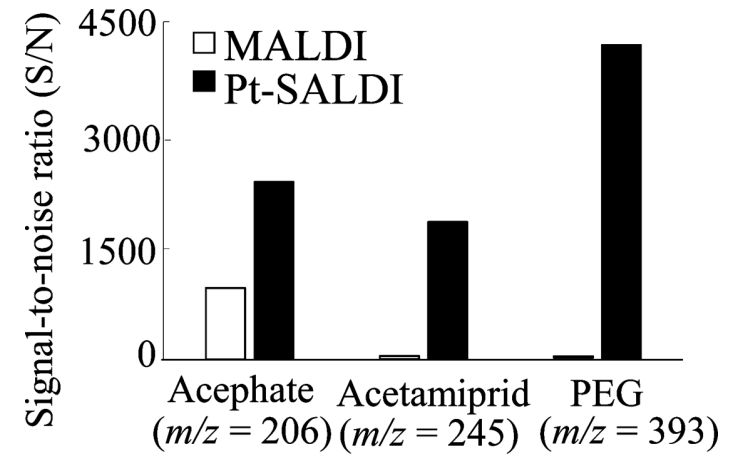

Fig. 2 Signal-to-noise ratios of acephate at $\mathrm{m} / \mathrm{z} 206$, acetamiprid at $\mathrm{m} / \mathrm{z}, 245$, and PEG at $\mathrm{m} / \mathrm{z}, 393$ on leaves using MALDI and Pt-SALDI.

thickness $(\sim 10 \mathrm{~nm})$ of the sputter-deposited Pt film with pores is important to produce high ion yields in Pt-SALDI; the $S / N$ of the molecular ions decreased with increasing thickness of the $\mathrm{Pt}$ film. ${ }^{27,28}$

IMS monitoring of absorption and migration of agricultural chemicals in plants

Direct IMS-analysis was demonstrated for the Pansy leaves spotted with standard agricultural chemicals (acephate and acetamiprid) at different concentrations using Pt-SALDI-IMS as 

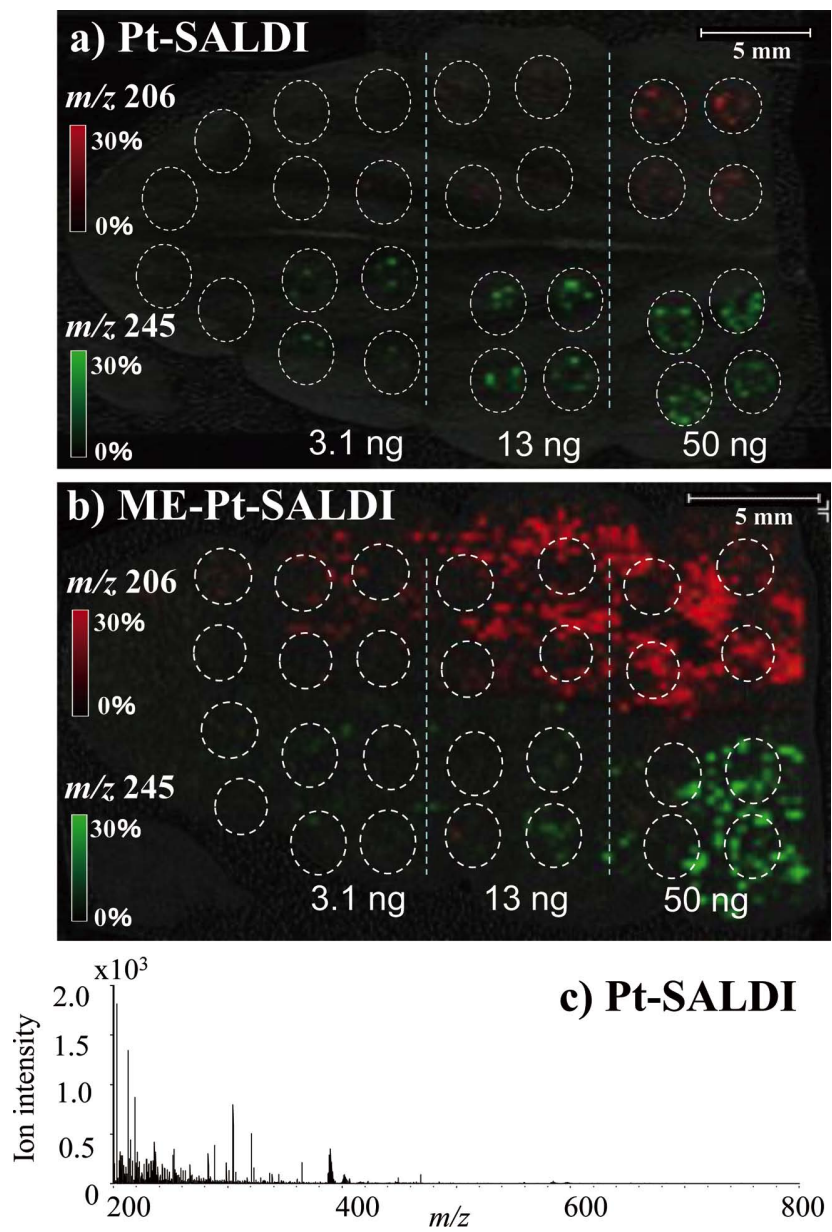

Fig. 3 IMS images of acephate at $\mathrm{m} / \mathrm{z}, 206$ and acetamiprid at $\mathrm{m} / \mathrm{z}$ 245 on leaves using a) Pt-SALDI, b) ME-Pt-SALDI, and c) Pt-SALDI spectrum of blank on leaf.

shown in Fig. 3a. In the Pt-SALDI-IMS technique, sodium adduct ions of acephate at $\mathrm{m} / \mathrm{z}, 206$ and acetamiprid at $\mathrm{m} / \mathrm{z}, 245$ were detected on the leaf. The distributions of acephate at $\mathrm{m} / \mathrm{z}$ 206 and acetamiprid at $\mathrm{m} / \mathrm{z}, 245$ were denoted by red color and green color in Fig. 3, respectively. In Fig. 3c, these chemicals were not observed. Acephate at a concentration of $50 \mathrm{ng}$ and acetamiprid at concentrations of $3.1,13$, and $50 \mathrm{ng}$ were clearly observed from the spots. Acephate spots with concentrations of 3.1 and $13 \mathrm{ng}$ were also detected to a lesser extent. In MALDIIMS, however, the $S / N$ of the acephate and acetamiprid ion peaks were extremely low compared to that obtained using PtSALDI since the charge-up effect caused low ion yields of the samples in MALDI. Additionally, MALDI-IMS suffers from the disadvantages of migration and spread of the analyte spots on the leaves during the sample spraying process in sample preparation for MALDI. Therefore, a skilled technique and empirical knowledge of sample preparation for MALDI-IMS are necessary. Heterogeneous co-crystallization of the matrix and sample molecules obtained from the matrix spraying process may cause low reproducibility in MALDI-IMS. To examine the migration effect on the leaves in the spraying process, the hybrid method combining MALDI and Pt-SALDI (i.e., matrixenhanced [ME]-Pt-SALDI-IMS) was used since MALDI-IMS could not give clear images of leaves. For the ME-Pt-SALDI analysis, a leaf treated with standard samples was prepared and sprayed with a DHB matrix; on the leaf sprayed with DHB,
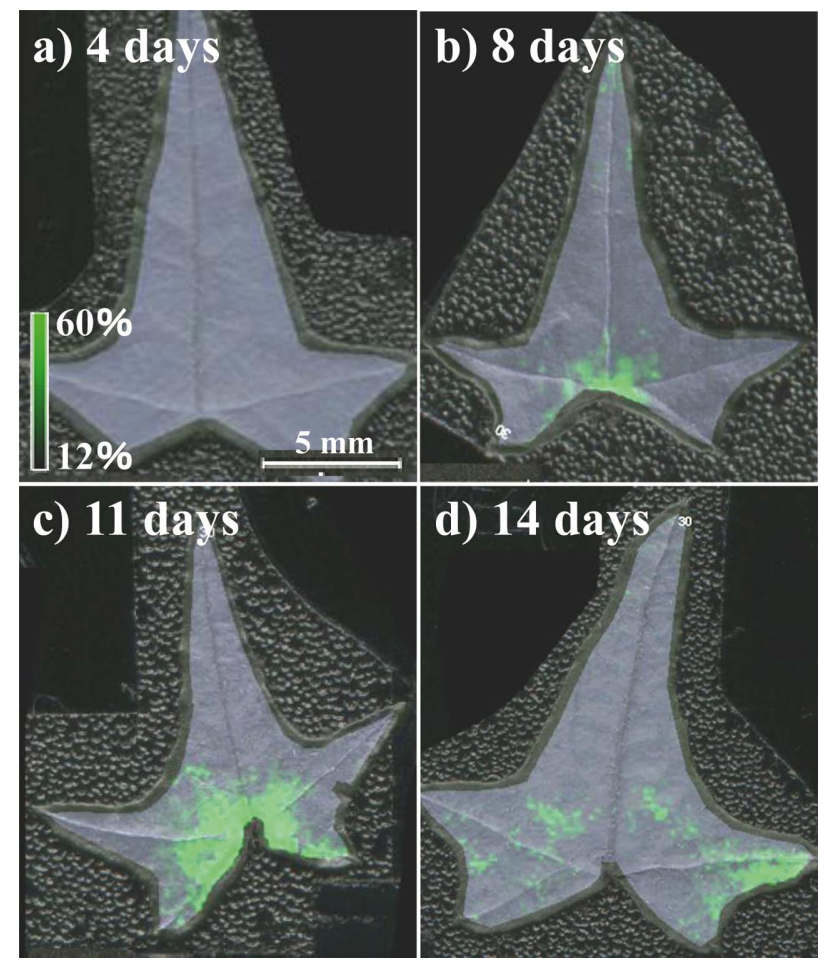

Fig. 4 Pt-SALDI-IMS images of acephate sodium adduct ion at $\mathrm{m} / \mathrm{z}$ 206 on leaves containing systemic pesticide GF Orutoran after a) 4, b) 8 , c) 11 , and d) 14 days.

the Pt film was deposited. The image obtained by the ME-PtSALDI is shown in Fig. 3b. The higher desorption efficiencies contributed from both MALDI and SALDI increased the strong signals of acephate and acetamiprid than those from SALDI only. ${ }^{28}$ The observed sample ions were migrated over the entire leaf. This result indicates that the spraying treatment used for MALDI-IMS was difficult and was not suitable for IMS of the leaves. The presented results indicate that solvent-free Pt-SALDI is a suitable method for direct analysis of leaves with the advantages of effective ionization, and imparting conductivity to the non-conductive sample surface, and not requiring a spraying matrix solution.

\section{IMS monitoring of distributions and movements of agricultural chemicals in plant}

Commercial agricultural chemical solutions contain solvent, surfactant, and minerals in addition to the pesticides. These compounds are added to permeate in the plant and to spread throughout the plant effectively. In this study, we analyzed the movement of systemic insecticides in soil into the plant using Pt-SALDI-IMS. The commercial agricultural pesticide "GF Orutoran" was utilized. GF Orutoran was mixed with soil, and its absorption and migration (i.e., permeability) from the prepared soil to the root of the plant and eventually into the leaves were analyzed by Pt-SALDI-IMS. Leaf samples were acquired after $4,8,11$, and 14 days by plucking the leaves from the stalk $30 \mathrm{~cm}$ away from the root. The leaves were coated by sputter-deposited Pt film immediately after picking, and the chemical distribution was measured by using Pt-SALDI-IMS.

Figure 4 shows Pt-SALDI-IMS images of the acephate sodium adduct ion at $\mathrm{m} / \mathrm{z}, 206$ merging scanned images of leaves for leaves treated with the systematic pesticide GF Orutoran after 4, 8,11 , and 14 days. Acephate, which is a component of the 
pesticide, was not detected in the image of the leaf after 4 days (Fig. 4a), indicating that acephate did not permeate into the leaf after 4 days. However, after 8 days, the leaves contained acephate near the stem side (Fig. 4b), and the acephate spread throughout the leaf after 11 days (Fig. 4c). In the image of the leaf after 14 days, the concentration of acephate at the stem side decreased and there was greater spread in the leaf (Fig. 4d). The sodium and potassium adduct ions of acephate were also detected in the leaf after 11 days. The distributions of the permeated agricultural chemicals in plants could be analyzed using Pt-SALDI-IMS via simple and easy Pt-sputtering preparation.

\section{Conclusions}

Standard samples of agricultural chemicals on leaves were analyzed using MALDI- and Pt-SALDI-IMS. These samples could not be adequately detected with MALDI-MS because of the charge-up effect and sample diffusion on the non-conductive leaf. Pt-SALDI gave higher $S / N$ for the samples than MALDI because of the conductive property of the leaf. The conductive property on leaves was imparted by the sputter-deposited Pt-film in the Pt-SALDI preparation. The distribution and movement of the components of the agricultural chemical GF Orutoran could be analyzed directly using Pt-SALDI-IMS. Changes in the properties of the chemicals in the plants with time, as an indicator of the permeability of the insecticides, could thus be observed. Pt-SALDI-IMS is therefore a useful tool for direct and rapid evaluation of the distributions of insecticides on leaves.

\section{Acknowledgements}

The authors acknowledge the support from the Creation of Innovation Centers for Advanced Interdisciplinary Research Area Program in the Special Coordination Fund for Promoting Science and Technology, and the support from the Grant-in-Aid for Scientific Research (C) (26505011) and Scientific Research (B) (15H03250) from the Japan Ministry of Education, Culture, Sports and Technology. This work was partly supported by the Nanotechnology Platform Program of the Ministry of Education, Culture, Sports, Science and Technology (MEXT), Japan.

\section{References}

1. R. Romeo-Gonzalez, A. G. Frenich, and J. L. M. Vidal, Talanta, 2008, 76, 211.

2. K. Greulich and L. Alder, Anal. Bioanal. Chem., 2008, 391, 183.

3. M. D. Gil-Garcia, D. Barranco-Martinez, M. MartinezGalera, and P. Parrilla-Vazquez, Rapid Commun. Mass Spectrom., 2006, 20, 2395.

4. K. Grossmann and T. Ehrhardt, Pest. Manag. Sci., 2007, 63, 429.
5. T. Müller, S. Oradu, D. R. Ifa, R. G. Cooks, and B. Kräutler, Anal. Chem., 2011, 83, 5754.

6. T. J. Garrett, M. C. Prieto-Conaway, V. Kovtoun, H. Bui, N. Izgarian, G. Stafford, and R. A. Yost, Intl. J. Mass Spectrom., 2007, 260, 166.

7. C. H. Le, J. Han, and C. H. Borchers, Anal. Chem., 2012, $84,8391$.

8. M. Karas, D. Bachmann, U. Bahr, and F. Hillenkamp, Int. J. Mass Spectrom. Ion Processes, 1987, 78, 53.

9. K. Tanaka, H. Waki, Y. Ido, S. Akita, and T. Yoshida, Rapid Commun. Mass Spectrom., 1988, 2, 151.

10. M. Karas and F. Hillenkamp, Anal. Chem., 1988, 60, 2299.

11. L. F. Marvin, M. A. Roberts, and L. B. Fay, Clin. Chim. Acta, 2003, 337, 11.

12. R. C. Murphy, J. A. Hankin, and R. M. Barkley, J. Lipid Res., 2009, 50, S317.

13. J. A. Hankin, R. M. Barkley, and R. C. Murphy, J. Am. Soc. Mass Spectrom., 2007, 18, 1646.

14. D. Cao, Z. Wang, C. Han, L. Cui, M. Hu, J. Wu, Y. Liu, Y. Cai, H. Wang, and Y. Kang, Talanta, 2011, 85, 345.

15. J. Sun and B. C. Lynn, J. Am. Soc. Mass Spectrom., 2007, 18, 698 .

16. A. Scherl, C. G. Zimmermann-Ivol, J. Di Dio, A. R. Vaezzadeh, P. A. Binz, M. Amez-Droz, R. Cochard, J. C. Sanchez, M. Glückmann, and D. F. Hochstrasser, Rapid Commun. Mass Spectrom., 2005, 19, 605.

17. P. A. Kuzema, J. Anal. Chem., 2011, 66, 1227.

18. C.-Y. Lo, J.-Y. Lin, W.-Y. Chen, C.-T. Chen, and Y.-C. Chen, J. Am. Soc. Mass Spectrom., 2008, 19, 1014.

19. J. Sunner, E. Dratz, and Y. C. Chen, Anal. Chem., 1995, 67, 4335.

20. Y. C. Chen, J. Shiea, and J. Sunner, J. Chromatogr., A, 1998, 826, 77.

21. M.-F. Huang and H.-T. Chang, Chem. Sci., 2012, 3, 2147.

22. C.-W. Wang, W.-T. Chen, and H.-T. Chang, J. Am. Soc. Mass Spectrom., 2014, 25(7), 1247.

23. C.-L. Su and W.-L. Tseng, Anal. Chem., 2007, 79(4), 1626.

24. J. A McLean, K. A Stumpo, and D. H. Russell, J. Am. Chem. Soc., 2005, 127(15), 5304.

25. T. C. Chiu, L. C. Chang, C. K. Chiang, and H. T. Chang, J. Am. Soc. Mass. Spectrom., 2008, 19, 1343.

26. H. Kawasaki, T. Yonezawa, T. Watanabe, and R. Arakawa, J. Phys. Chem. C, 2007, 111, 16278.

27. H. Kawasaki, T. Ozawa, H. Hisatomi, and R. Arakawa, Rapid Commun. Mass Spectrom., 2012, 26, 1849.

28. T. Ozawa, I. Osaka, T. Ihozaki, S. Hamada, Y. Kuroda, T. Murakami, A. Miyazato, H. Kawasaki, and R. Arakawa, $J$. Mass Spectrom., 2015, 50, 1264.

29. M. Dufresne, A. Thomas, J. Breault-Turcot, J. Masson, and P. Chaurand, Anal. Chem., 2013, 19, 3318.

30. L. Muller, A. Kailas, S. N. Jackson, A. Roux, D. C. Barbacci, J. A. Schultz, C. D. Balaban, and A. S. Woods, Int. Soc. Nephrology, 2015, doi:10.1038/ki.2015.3.

31. J. Grassl, N. L Taylor, and A. Millar, Plant Methods, 2011, 7, 21.

32. A. Liu and L. He, J. Am. Soc. Mass. Spectrom., 2009, 20, 2229. 